\title{
Quasiparticle Dynamics in Graphene
}

Aaron Bostwick, ${ }^{1}$ Taisuke Ohta,,${ }^{1,2}$ Thomas Seyller, ${ }^{3}$ K. Horn ${ }^{2}$ and Eli Rotenberg ${ }^{1}$

${ }^{1}$ Advanced Light Source, E. O. Lawrence Berkeley National Laboratory, Berkeley, CA 94720 USA

${ }^{2}$ Department of Molecular Physics, Fritz Haber Institute, Germany

${ }^{3}$ Institut für Physik der Kondensierten Materie, Universität Erlangen-Nürnberg, Germany

The effectively massless, relativistic behaviour of graphene's charge carriers - known as Dirac fermions

- is a result of its unique electronic structure, characterized by conical valence and conduction bands

that meet at a single point in momentum space (at the Dirac crossing energy). The study of many-body

interactions amongst the charge carriers in graphene and related systems such as carbon nanotubes, fullerenes and graphite is of interest owing to their contribution to superconductivity and other exotic ground states in these systems. Here we show, using angle-resolved photoemission spectroscopy (ARPES), that electron-plasmon coupling plays an unusually strong role in renormalizing the bands around the Dirac crossing energy - analogous to mass renormalization by electron-boson coupling in ordinary metals. Our results show that electron-electron, electron-plasmon and electron-phonon coupling must be considered on an equal footing in attempts to understand the dynamics of quasiparticles in graphene and related systems.

With the recent discovery of superconductivity in carbon nanotubes $(\mathrm{CNTs}),{ }^{1,2}$ alkaline-metal-doped $\mathrm{C}_{60}$

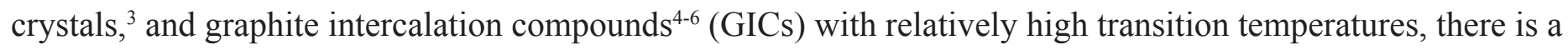
strong interest in the influence of many-body interactions on the electron dynamics in these systems. Graphene is a sheet of carbon atoms distributed in a honeycomb lattice and is the building block for all of these materials; therefore it is a model system for this entire family. Recently, graphene has been isolated using exfoliation from graphite ${ }^{7,8}$ and graphitization of $\mathrm{SiC},{ }^{9,10}$ enabling, for the first time, the direct measurement of the manybody interactions fundamental to all of these carbon systems. These interactions could be especially interesting owing to the effectively massless, relativistic nature of the charge carriers, which follows from the linearity of the bands at the Dirac crossing energy $E_{\mathrm{D}}=\hbar \omega_{\mathrm{D}}$ and the formal equivalence of the Schrödinger wave equation with the relativistic Dirac equation for graphene. $7,8,11$ 
The coupling among quasiparticles is fundamental to understanding superconductivity and other exotic ground states. We focus in particular on the interaction of the carriers with electron-hole pair excitations within the Fermi-liquid model, and with plasmons. Departure of the electron dynamics from Fermi liquid behavior in graphite has already been attributed to the special shape of the graphene bandstructure, ${ }^{12,13}$ while electronplasmon scattering has been proposed as a key coupling process in superconductivity ${ }^{14}$ of cuprates ${ }^{15}$, and possibly metallized graphene. ${ }^{16}$ ARPES probes the scattering rate at different energy scales, and therefore accesses these many-body couplings directly. We find that both electron-hole and electron-plasmon effects are important for graphene, depending on the energy scale, and, together with electron-phonon coupling, are necessary for a complete picture of the quasiparticle dynamics.

The single-particle graphene bandstructure $E(\mathbf{k})$ may be described by a simple one-orbital tight-binding model as ${ }^{17}$

$$
E(\mathbf{k})= \pm t \sqrt{1+4 \cos \left(\sqrt{3} a k_{y} / 2\right) \cos \left(a k_{x} / 2\right)+4 \cos ^{2}\left(a k_{x} / 2\right)}
$$

where $\mathbf{k}$ is the in-plane momentum, $a$ is the lattice constant, and $t$ is the near-neighbor hopping energy. That the carriers travel as effectively massless particles with a fixed "speed of light" $c^{*}=\hbar^{-1} d E(k) / d k \sim c / 300$ follows from the nearly linear dispersion of the bands at zero energy $\left(E_{\mathrm{D}}\right)$. In Figure 1 we compare energy bands and constant energy surfaces computed using Equation 1 to the first (to our knowledge) ARPES measurements applied to a single layer of graphene, grown on the (0001) surface of $\mathrm{SiC}(6 \mathrm{H}$ polytype). (Previous ARPES measurements near $E_{\mathrm{F}}$ have focused on thicker graphene layers on $\mathrm{SiC}^{18-20}$ or on bulk graphite. ${ }^{21,22}$ ) The primary bands, cones centered at the K points, are surrounded by six weak replica bands discussed further below. The primary bands are in good overall agreement with the simple model despite its having only two adjustable parameters: the hopping energy $t=2.82 \mathrm{eV}$ and a $0.435 \mathrm{eV}$ shift of the Fermi energy $E_{\mathrm{F}}$ above the Dirac crossing energy $E_{\mathrm{D}}$. This shift is attributed to doping of the graphene layer by depletion of electrons from the $n$-type SiC.

We can discriminate a single layer of graphene from thicker films by counting the number of $\pi$ states (one in Figure 1), which is equal to the number of layers $m$ in a given sample. ${ }^{20}$ While films of thickness $m$ $\geq 2$ often coexist with $(m \pm 1)$-layer domains, we found that pure graphene films $(m=1)$ can be routinely isolated. We can also be sure that there is negligible interaction between overlayer and substrate states. This follows naturally from the fact that the Fermi level of the graphene is pinned well within the $\sim 3 \mathrm{eV}$ band gap 
of the substrate. If there were hybridization with the substrate, we would expect it not near $E_{\mathrm{F}}$ but rather at a deeper binding energy, where the substrate and graphene bands overlap. Even there, we see no indication of interactions between the graphene and substrate band structures in Figure 1.

Such interactions are not expected considering the proposed van der Waals bonding between graphene and $\mathrm{SiC}^{9}$. Recent experiments have shown that the SiC layer immediately below the graphene is itself a carbon-rich layer, with an in-plane, graphene-like network of $s p^{2}$-derived $\sigma$ bands, but without graphene-like $\pi$ bands ${ }^{23}$. The absence of states at the Fermi level suggests that the $p_{z}$ orbitals are saturated, presumably due to bonding with the substrate as well as bonding within the C-rich interface layer. This C-rich layer is a perfect template for van der Waals bonding to the overlying graphene because it offers no $p_{z}$ orbitals for bonding to the graphene. The photon energy dependence of the $\pi$-band intensities, absent for $m=1$ films, but clearly observed for $m \geq 2$, confirms this lack of hybridization [Ohta, Bostwick, McChesney, Seyller, Horn, Rotenberg, in preparation].

The only effect of the interface on the measurements is through the nearly incommensurate $(6 \sqrt{3} \times 6 \sqrt{3}) \mathrm{R} 30^{\circ}$ symmetry of the interface C-rich layer with respect to SiC. This interface induces diffraction of the primary bands, resulting in the observed weak satellite bands, similar to the satellite spots seen in low energy electron diffraction. ${ }^{9}$

Despite the overall good agreement between Equation 1 and the data in Figure 1, profound deviations are observed when we examine the region around $E_{\mathrm{F}}$ and $E_{\mathrm{D}}$ in more detail. Figure 2a shows a magnified view of the bands measured along a line (the vertical double arrow in Figure 1b) through the K point. The predicted, or "bare" bands in this direction are nearly perfectly linear and mirror-symmetric with respect to the K point according to Equation 1, similar to the H point of bulk graphite. ${ }^{21,22}$ The actual bands deviate from this prediction in two significant ways: First, at a binding energy $\hbar \omega_{\mathrm{ph}} \sim 200 \mathrm{meV}$ below $E_{\mathrm{F}}$, we observe a sharpening of the bands accompanied by a slight kink in the bands' dispersions. We attribute this feature to renormalization of the electron bands near $E_{\mathrm{F}}$ by coupling to phonons, ${ }^{24}$ as discussed later.

Second, and more surprisingly, linear extrapolations of the lower bands (dashed lines in Figure 2a) do not pass through the upper bands, demonstrating that the bands do not pass smoothly through $E_{\mathrm{D}}$ as Equation 1 predicts. This is observed more easily for data acquired along the orthogonal direction through the K point (Figure 2e), along which an interference effect ${ }^{25}$ suppresses one of the two bands. We see that near $E_{\mathrm{D}}$ the bands 
have an additional kink, which we propose is caused by other many-body interactions.

The deviations from the bare band are sensitive to doping, which was varied by adsorbing potassium atoms that readily donate electrons to the graphene. The evolution of the band structure with increasing doping is followed in Figure $2 \mathrm{~b}-\mathrm{d}$ and along the orthogonal direction in Figure $2 \mathrm{f}-\mathrm{h}$. Similar to graphite, doping graphene by $\mathrm{K}$ deposition shifts the bands more or less rigidly to higher binding energy. ${ }^{26}$ While the energy of the kink at $200 \mathrm{meV}$ does not change, the deeper-energy kink strengthens and follows $E_{\mathrm{D}}$ with doping, demonstrating that it is associated with electrons with energy near $E_{\mathrm{D}}$. The effect of this kink on the bandstructure is significant: at high doping, a curve fit of the band positions (small circles in Figure 2d) shows that $E_{\mathrm{D}}$ has been shifted towards $E_{\mathrm{F}}$ by $\sim 130 \mathrm{meV}$ from the single-particle prediction.

In the quasiparticle scheme, the carriers are represented as single particles that scatter from, and are surrounded by, a cloud of other 'particles' (such as phonons); the entire entity moves somewhat like a free particle but with renormalized energy. In this scheme, ARPES measures the spectral function, expressed in terms of the complex self-energy $\Sigma(\mathbf{k}, \omega)$, as

$$
A(\mathbf{k}, \omega)=\frac{|\operatorname{Im} \Sigma(\mathbf{k}, \omega)|}{\left(\omega-\omega_{\mathrm{b}}(\mathbf{k})-\operatorname{Re} \Sigma(\mathbf{k}, \omega)\right)^{2}+(\operatorname{Im} \Sigma(\mathbf{k}, \omega))^{2}}
$$

where $\mathbf{k}$ and $\omega$ are the momentum and energy, and $\omega_{\mathrm{b}}$ is the bare band dispersion in the absence of many-body effects. $\Sigma(\mathbf{k}, \omega)$ contains both the scattering rate and the renormalization of the band dispersion in its imaginary and real parts, respectively. In the k-independent approximation ${ }^{27,28}(\Sigma(\mathbf{k}, \omega) \approx \Sigma(\omega)), \operatorname{Im} \Sigma(\omega)$ is proportional to the Lorentzian linewidth of the momentum distribution curve (MDC) $A(\mathbf{k}, \omega)$ taken at constant $\omega . \operatorname{Re} \Sigma(\omega)$ is readily computed from $\operatorname{Im} \Sigma(\omega)$ through a Hilbert transform (to satisfy causality), and the full spectral function $A(\mathbf{k}, \omega)$ can be reconstructed using the computed $\operatorname{Re} \Sigma(\omega)$ and compared to experiment. Such a reconstruction for one doping is shown in Figure 2i; it is in excellent agreement with the data (Figure $2 \mathrm{~h}$ ) from which $\operatorname{Im} \Sigma$ was obtained. This shows that all the kinks in the bands originate not from details of the single-particle bandstructure, but rather from many-body interactions, providing strong support for the quasiparticle picture in graphene.

The observed kink structure is therefore derived from a complicated $\omega$-dependence of the observed scattering rate proportional to the measured MDC linewidths, shown in Figure 3 as a function of doping. To model these data, we consider three processes: decay of the carriers by electron-phonon (e-ph) coupling, by 
electron-hole $(e-h)$ pair generation, and by emission of collective charge excitations (plasmons) via electronplasmon $(e-p l)$ coupling. (Impurity scattering, a fourth contributing process, can be neglected as its contribution to the MDC linewidth is smaller than the experimental momentum resolution $\left(\sim 0.01 \AA^{-1}\right)$ and in any case merely leads to a uniform background scattering rate). By summing up all the momentum- and energy-conserving decay events as a function of hole energy $\omega$, we can show that the three principal decay processes $(e-h, e-p l$, and $e-p h)$ contribute differently to the lifetime in regions I-IV as identified in the traces at the upper part of Figure 3, calculated for a sample with $n=5.6 \times 10^{13} \mathrm{~cm}^{-2}$ and compares favorably to the experimental MDC width for that doping; similar agreement can be obtained for the other dopings as well. (The predicted dip at $E_{\mathrm{D}}$ is an artifact of the simplicity of our model, which does not consider interactions between the plasmons and the Fermi liquid excitations.)

Now we discuss the different decay processes in turn. We attribute the kink near $E_{\mathrm{F}}$ to $e$-ph coupling as described previously for metals, ${ }^{29-31}$ for (possibly) high- $T_{\mathrm{c}}$ superconductors ${ }^{32,33}$ and for bulk graphite. ${ }^{24}$ In this process, photoholes decay by phonon emission (see Figure 4a). From graphite's phonon density of states, ${ }^{34}$ we calculated the $e-p h$ contribution to $\operatorname{Im} \Sigma$ (Figure 3, green curve) with the standard formalism ${ }^{35}$ and find an $e-p h$ coupling constant $\lambda \approx 0.3$. Although this is a factor of 5 larger than predicted ${ }^{36}$ for $n=5.6 \times 10^{13} \mathrm{~cm}^{-2}$, comparison with the experimental data shows that this provides an accurate description of $\operatorname{Im} \Sigma$ in region I. The observed increase of the kink's strength with $n$ (see Figure 2e-h) is expected from the increase in the size of the Fermi surface, although the $200 \mathrm{meV}$ energy scale remains constant because the $\mathrm{K}$ atoms should not alter the phonon bandstructure at this energy.

Consider now the decay of the photohole by excitation of an electron from below to above $E_{\mathrm{F}}$, thereby creating an $e-h$ pair. In Landau's Fermi Liquid (FL) theory, the scattering rate from such processes increases as $\sim \omega^{2}$ away from $\omega=0$, reflecting the growing number of possible excitations that satisfy momentum and energy conservation. However, the linear dispersion of the graphene bands and the presence of the Dirac crossing below $E_{\mathrm{F}}$ drastically modify this picture. ${ }^{37}$ A hole just above $E_{\mathrm{D}}$ can easily decay through many possible $e-h$ creation events, for example as in Figure 4b, and we find a $\omega^{\alpha}(\alpha \sim 1.5)$ dependence of $\operatorname{Im} \Sigma$ in regions I-II in contrast with FL theory $(\alpha=2)$. But a hole originating at $\omega$ just below $E_{\mathrm{D}}$ has few possible decays with sufficient momentum transfer to excite an $e-h$ pair (Figure 4c). This causes a sharp reduction in the scattering rate in region III, seen in the red curve in Figure 3. Only for energies $\omega \lesssim 2 \omega_{\mathrm{D}}$, region IV, does $e$ - $h$ pair generation become favorable (e.g. Figure 4d). 
The $e-h$ and $e-p h$ processes can explain the observed MDC widths in regions I, II, and IV. In region III, however, decay by $e-h$ pair creation is practically not allowed yet the observed scattering rate has a peak rather than a dip (highlighted in blue in Figure 3). We now show that this peak may be explained by decay through plasmon emission. Plasmons are oscillations of an electron gas that play an important role in the optical properties of ordinary metals. In graphene, the charge carriers near the $\mathrm{K}$ point have zero effective mass and travel like photons at constant speed $c^{*}$, but unlike photons, they have charge and are therefore subject to collective oscillations such as plasmons. Although a full treatment of the $e$ - $p l$ interaction is difficult near the Dirac point, a simple model suffices to explain how e-pl coupling can enhance the scattering rate below $E_{\mathrm{D}}$.

Ordinary two-dimensional plasmons have a dispersion relationship

$$
\omega_{\mathrm{pl}}(q)=\sqrt{4 \pi n e^{2} q / m(1+\varepsilon)}
$$

where $q$ is the plasmon momentum, $m$ is the effective carrier mass, and $\varepsilon$ is the dielectric constant. For graphene, the rest mass $m_{0}$ is zero near $E_{\mathrm{D}}$, but the "relativistic mass" $m_{\mathrm{r}}=E / c^{* 2}$ depends on the doping," ${ }^{8}$ reaching a maximum of only $10 \%$ of the free electron rest mass for our samples; this has a dramatic effect to increase the plasmon energy $\omega_{\mathrm{pl}}(q)$ as shown in Figure 5, calculated for a reasonable range $(3<\varepsilon<10)$ of dielectric constants.

Collective plasmon excitations are not really independent of the $e-h$ pair excitations discussed above, and therefore decay by plasmon scattering is only a valid description outside the range of kinematically allowed $e-h$ processes, as shown in Figure 5 for $n$-doped graphene. This occurs only for decay processes with $\omega \sim E_{\mathrm{D}}$ and $q \sim 0$ (as in Figure 4e), when the plasmon spectrum does not overlap the continuum of $e$ - $h$ excitations (blue region in Figure 5). This means that plasmons can have a large effect on the self energy around $\omega \sim \omega_{D}$.

Given the plasmon dispersion relation we can easily sum up the possible plasmon decays as a function of $\omega$ (Figure 3, blue curve), which is proportional to the scattering rate. We find a peak in $\operatorname{Im} \Sigma$ located just below $E_{\mathrm{D}}$, whose width and intensity scales with $E_{\mathrm{D}}$. A peak following these trends is clearly observed in the experimental data (highlighted in blue in Figure 3).

Previously, $e-p l$ coupling was shown to affect the unoccupied bands of a 3-dimensional metal at the large plasmon energy scale $(\sim 20 \mathrm{eV})^{38}$ But $e$ - $p l$ coupling at small energy scales is normally forbidden for two- and 
three-dimensional electron gases (except for the special case of layered electron gase ${ }^{39}$ ) so this is a unique instance where $e-p l$ coupling is kinematically allowed for a pure 2D system.

It is worth emphasizing that the model for the scattering rate has only four adjustable scaling factors: the $e$-ph coupling constant $\lambda$, the absolute probabilities for $e-h$ pair creation and plasmon emission, and the screening constant $\varepsilon$ which scales the Coulomb interaction. The other inputs are the experimentally determined band dispersion, the graphite phonon density of states, ${ }^{34}$ and the relativistic mass ${ }^{7,8} m_{\mathrm{r}}$.

These results show that the special condition of massless Dirac Fermions found in graphene does not preclude the validity of the quasiparticle picture - in fact the quasiparticle picture is valid over a spectacularly wide energy range - but it does induce novel $e-h$ and $e-p l$ decay processes. Thus, it is an exceptional combination of effects- the unusual bandstructure of graphene (leading to a large hole in the phase space for $e-h$ pairs) together with the small effective mass of the carriers (leading to a plasmon spectrum that fills this hole) that lead to these novel effects. These result in strong modifications of the band dispersion, as schematically illustrated in Figure 4f. This distortion occurs not only near the Fermi level as in conventional metals, but also centered around the Dirac crossing energy $E_{\mathrm{D}}$. The effects we describe are not unique to high doping levels, but extrapolate all the way down to zero doping. Near this regime (already approached for the lowest dopings in Figure $3 \mathrm{~b}$ ), the energy scales for $e-h, e-p l$, and $e-p h$ decay processes overlap, and a unified treatment of all these interactions is necessary to reproduce the many-body effects. These conclusions might apply as well to graphite, which also shows a 2D Dirac spectrum, ${ }^{40}$ metallic carbon nanotubes, which have a similar gapless band crossing ${ }^{41}$ and Dirac fermions, ${ }^{42}$ and other materials with similar electronic structure. ${ }^{37}$

Acknowledgements . This work and the ALS were supported by the Department of Energy, Office of Basic Sciences. K. H. and T. O. were supported by the Max Planck Society. We are grateful to J. L. McChesney for discussions and assistance with the experiments.

Author Contributions. T. S. and K. H. prepared the SiC substrates. T. O. optimized the graphene quality with help from A. B.; A. B. and T. O. contributed equally to the graphetization during data runs and ARPES measurements. A. B. performed theoretical modelling. E. R. performed numerical analysis of the data. E. R. and $A$. B. wrote the text with review and input from all other coauthors. 
Author Information. The authors declare no competing financial interests.

\section{Methods.}

The ARPES measurements were conducted at the Electronic Structure Factory endstation at beamline 7.01 at the Advanced Light Source, equipped with a hemispherical Scienta R4000 electron analyzer. The single layer of graphene was prepared by etching a $6 \mathrm{H}-\mathrm{SiC}(0001)$ substrate ( $n$-type with a nitrogen concentration of $(1.5 \pm 0.5) \times 10^{18} \mathrm{~cm}^{-3}$ from SiCrystal $\left.\mathrm{AG}\right)$ in hydrogen $\left(\mathrm{p}=1\right.$ bar, $\left.\mathrm{T}=1550^{\circ} \mathrm{C}, \mathrm{t}=30 \mathrm{~min}\right)$ followed by annealing at $1150 \mathrm{C}$ for 4 minutes by direct current heating in pressure better than $1 \times 10^{-10} \mathrm{Torr}^{9}, 10$. Measurements were conducted at a pressure better than $2.5 \times 10^{-11}$ Torr with the sample cooled to $\sim 20 \mathrm{~K}$ using a photon energy $h v$ of $95 \mathrm{eV}$ and with an overall energy resolution of $\sim 25 \mathrm{meV}$. Potassium deposition was by a commercial (SAES) getter source. The potassium coverage can be estimated from the carrier density assuming a charge transfer of $0.7 \mathrm{e}^{-}$per alkali atom ${ }^{43}$ to be about 0.007 monolayers when $n=1 \times 10^{13} \mathrm{~cm}^{-2}$.

The range of dielectric constants $(3<\varepsilon<10)$ used in the model is a rough estimate based on the dielectric constants of graphite (14), silicon carbide (10) and the vacuum (1). 

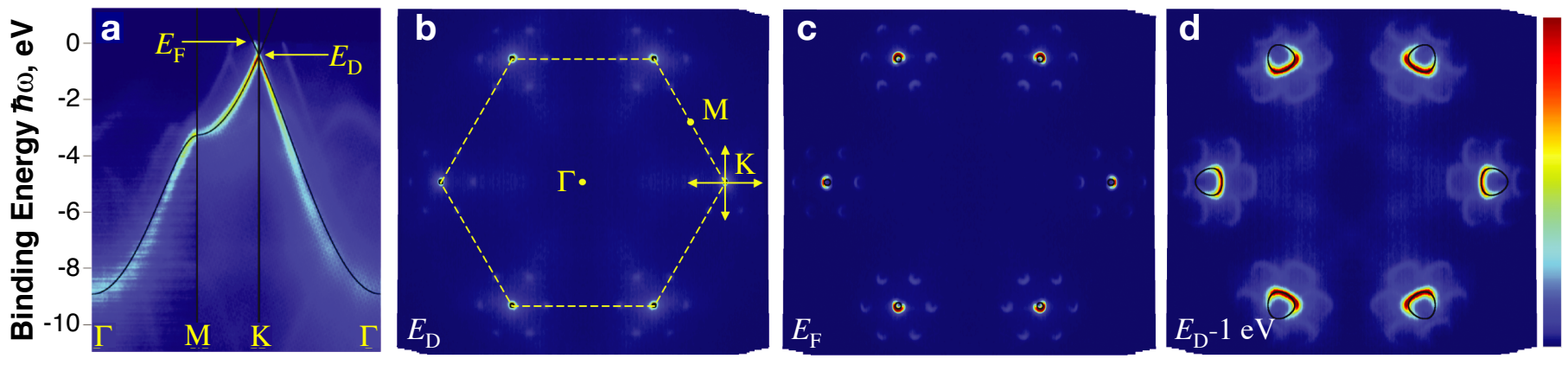

Momentum

Figure 1 | The bandstructure of graphene. a The experimental energy distribution of states as a function of momentum along principal directions, together with a single-orbital model (solid lines) given by Eq. (1). b Constant energy map of the states at binding energy corresponding to the Dirac energy $\left(E_{\mathrm{D}}\right)$ together with the Brillouin Zone boundary (dashed line). The orthogonal double arrows indicate the 2 directions over which the data in Fig. 2 were acquired. c-d Constant energy maps at the Fermi energy $\left(E_{\mathrm{F}}=E_{\mathrm{D}}+0.45\right)$ and $E_{\mathrm{D}}-1.5 \mathrm{eV}$, respectively. The faint replica bands correspond to the $6 \sqrt{ } 3 \times 6 \sqrt{3}$ satellite peaks in low energy electron diffraction. ${ }^{9}$ 

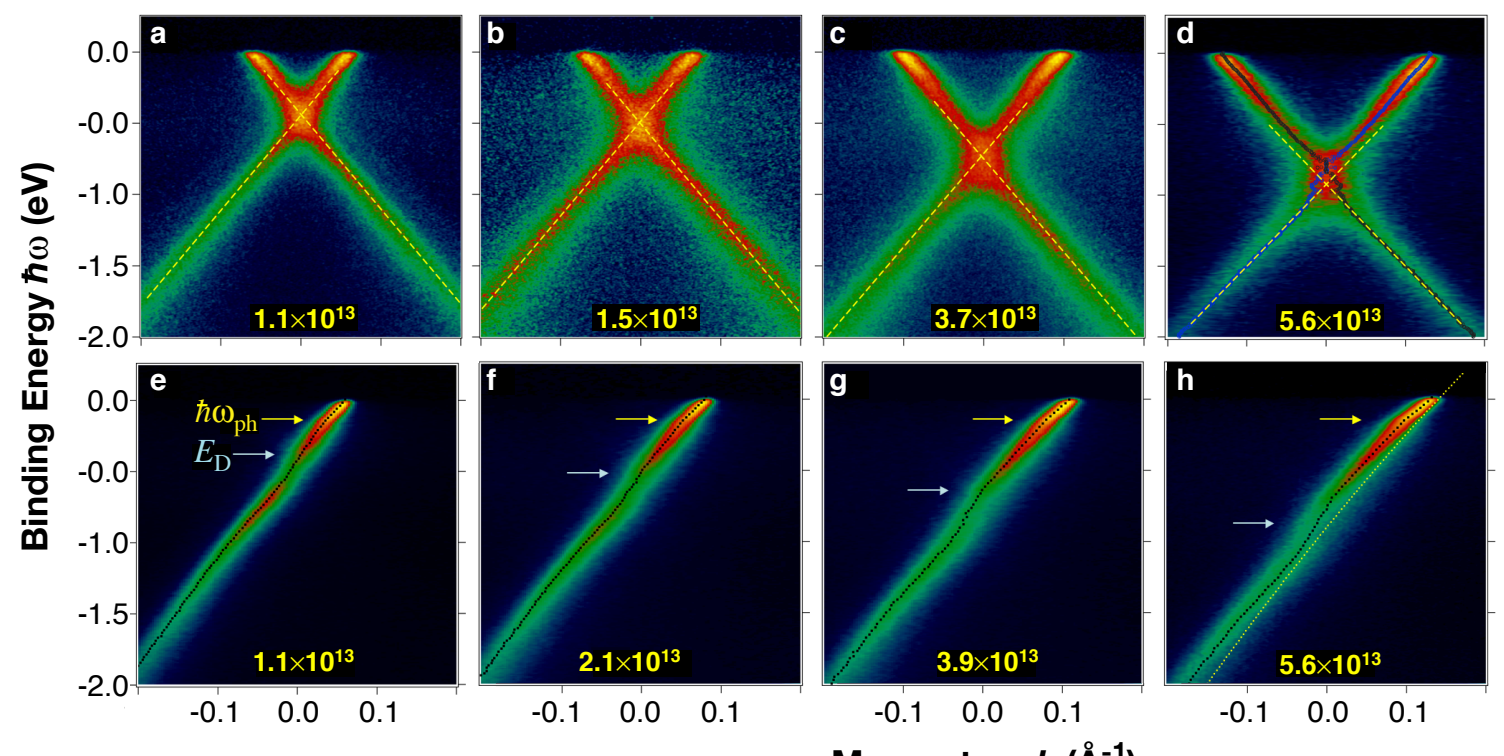

$\begin{array}{lll}-0.1 & 0.0 & 0.1\end{array}$

$\begin{array}{lll}-0.1 & 0.0 & 0.1\end{array}$

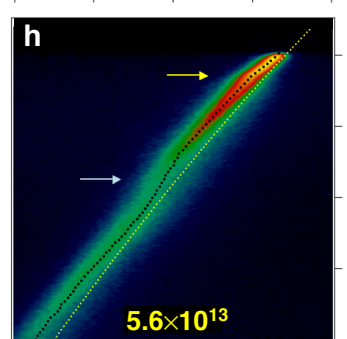

$\begin{array}{lll}-0.1 & 0.0 & 0.1\end{array}$

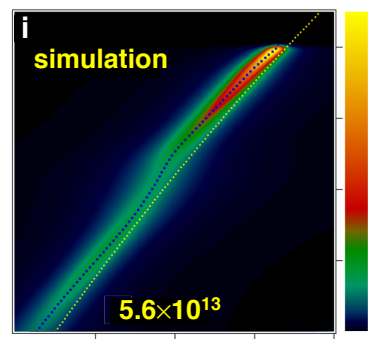

$\begin{array}{lll}-0.1 & 0.0 & 0.1\end{array}$

Momentum $k\left(\AA^{-1}\right)$

Figure 2 | The bandstructure of graphene near the Fermi Level. a-d Experimental energy bands along a line through the $\mathrm{K}$ point parallel to $\mathrm{\Gamma M}$ direction (along the vertical double-arrow in Figure 1b) as a function of progressively increased doping by potassium adsorption. The dashed lines are an extrapolation of the lower bands (below the Dirac crossing energy $E_{\mathrm{D}}$ ), which are observed not to pass through the upper bands (above $E_{\mathrm{D}}$ ), suggesting the kinked shape of the bands around $E_{\mathrm{D}}$. The electron density $\left(\right.$ per $\left.\mathrm{cm}^{2}\right)$ is indicated on each panel. e-h Bandmaps for similar dopings acquired in an orthogonal direction through the $\mathrm{K}$ point (horizontal double arrow in Figure 1b), for which one of the bands is suppressed. The non-linear, or "kinked" dispersion of the bands together with linewidth variations (corresponding to $\operatorname{Re}$ and $\operatorname{Im}$ parts of the self energy $\Sigma$ ) are clearly visible in the fitted peak positions (dotted lines). The kinks, marked by arrows, occur at a fixed energy of $200 \mathrm{meV}$ and near $E_{\mathrm{D}}$, the latter varying with doping. $\mathbf{i}$ The simulated spectral function, calculated using only the bare band (yellow dotted line) and $\operatorname{Im} \Sigma$ derived from the data in panel $\mathrm{h}$. 


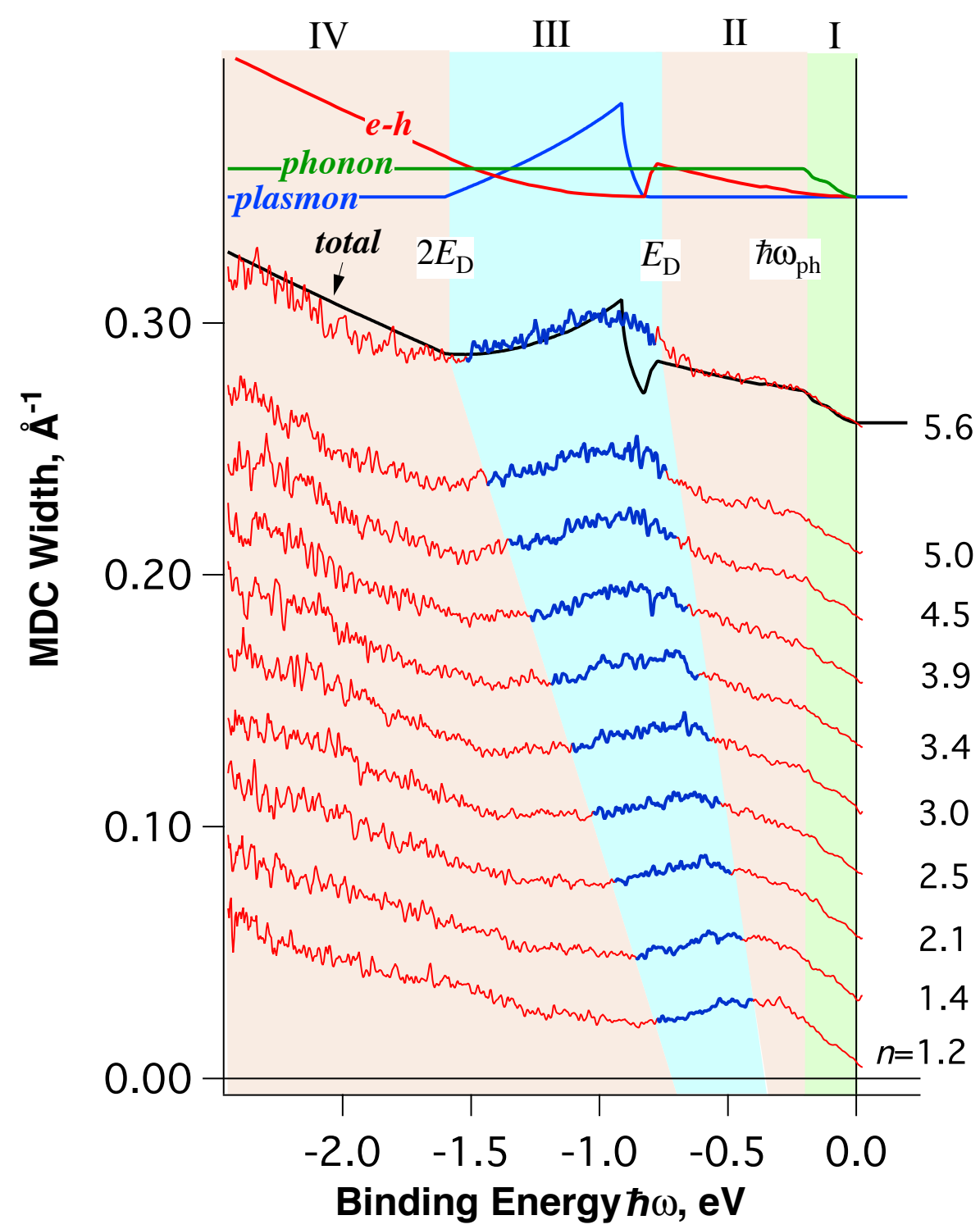

Figure 3 | Momentum Distribution Curve Widths of Carriers in Graphene. Measured spectral MDC width (assumed proportional to scattering rate and $\operatorname{Im} \Sigma$ ) for graphene, derived by performing a line shape analysis of momentum distribution curves for each binding energy as a function of doping $n$ (in units $10^{13} \mathrm{~cm}^{-2}$ ). Each trace is shifted upward by $0.025 \AA^{-1}$. The simulated total scattering rate (black line) and the partial contributions due to decay into phonons (green), electron-hole pairs (red) and plasmons (blue) are compared to the MDC spectral width for the highest doped sample. The plasmon calculation was for $\varepsilon=10$. These interactions contribute differently in regions I-IV defined as follows: (I) the phonon energy scale $\omega_{\mathrm{ph}}<\omega<0$, (II) the Dirac energy scale $\omega_{\mathrm{D}}<\omega<0$, (III) $2 \omega_{\mathrm{D}}<\omega$ $<\omega_{\mathrm{D}},(\mathrm{IV}) \omega<2 \omega_{\mathrm{D}}$. 

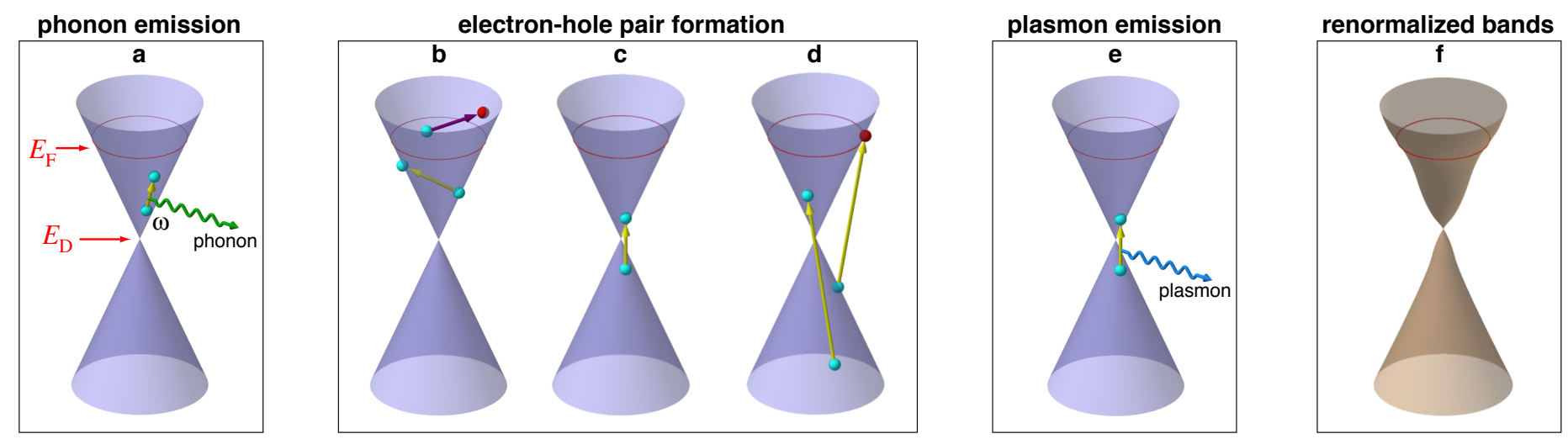

Figure 4 | Decay and Scattering Processes in Graphene. a The energy/momentum diagrams for decay processes scattering of a photohole, initially created at energy $\omega$, decaying by a emission of a phonon. b-d Spontaneous generation of an electron-hole pair near the Fermi level $E_{\mathrm{F}}$ for photohole energy satisfying $\mathbf{b}\left(\omega>\omega_{D}\right), \mathbf{c}\left(2 \omega_{D}<\omega<\omega_{D}\right)$, which can generate no possible $e-h$ pair as drawn, and $\mathbf{d}\left(\omega<2 \omega_{\mathrm{D}}\right)$. e emission of a plasmon $\left(2 \omega_{\mathrm{D}}<\omega<\omega_{\mathrm{D}}\right)$. $\mathbf{f}$ The net effect of these processes is to distort the bare bands to the renormalized bands (shown in brown). 


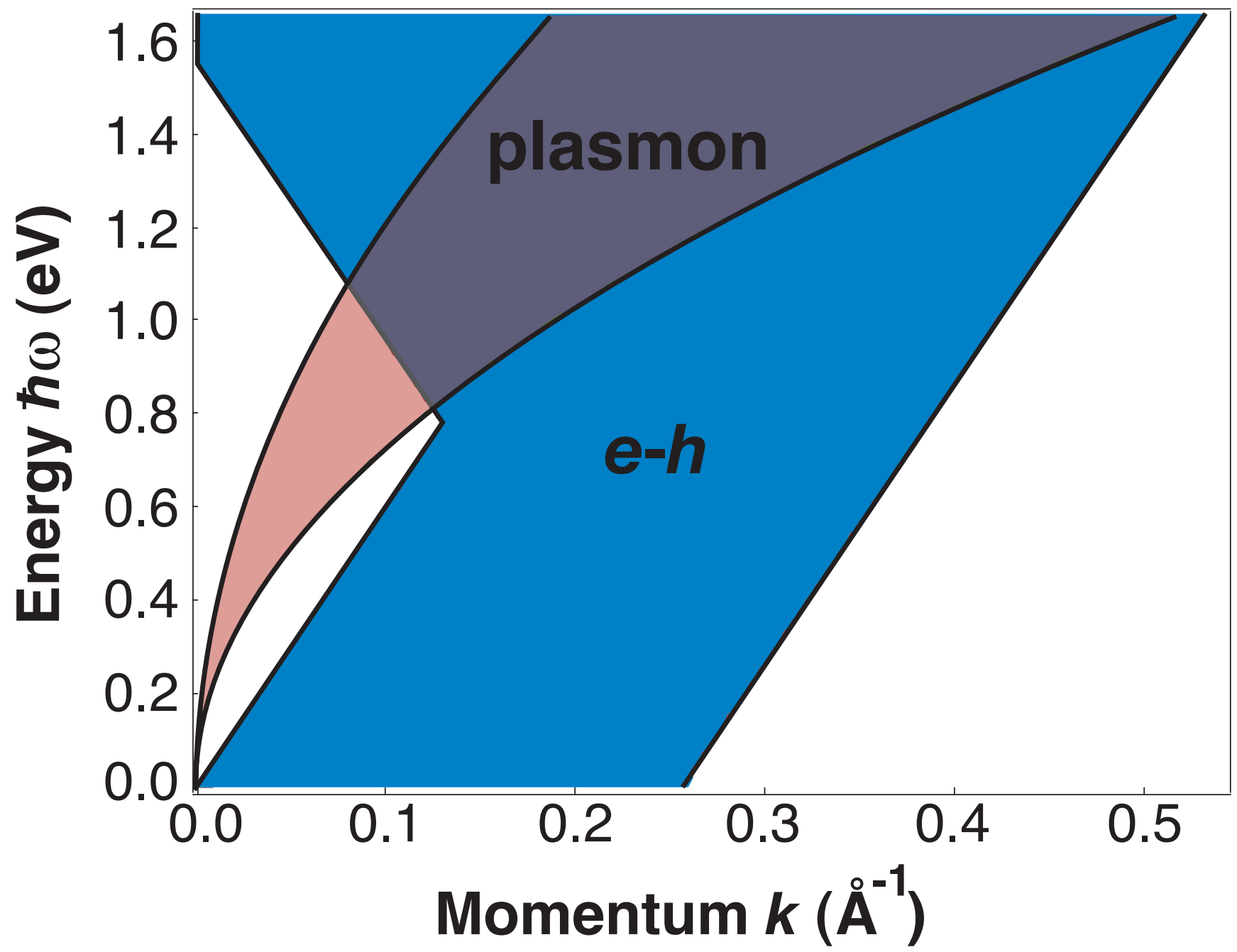

Figure 5 | Energy Diagram of Electronic Excitations in Graphene. The blue region shows the possible $e-h$ pair excitations for graphene, computed for a conical bandstructure with $n=5.6 \times 10^{13} \mathrm{~cm}^{-}$ ${ }^{2}$ as in Figure $4 a$. The pink region shows the plasmon dispersion calculated for a range of dielectric constants from $\varepsilon=3$ (upper rim) to $\varepsilon=10$ (lower rim) using Equation 3 together with the relativistic mass taken from transport measurements ${ }^{7,8}$. The e-p/ decay process occurs only for plasmons outside the blue region, where plasmons are well-defined quasiparticles. 
1. Tang, Z. K. et al. Superconductivity in 4 Angstrom Single-Walled Carbon Nanotubes. Science 292, 2462-5 (2001).

2. Kociak, M. et al. Superconductivity in Ropes of Single-Walled Carbon Nanotubes. Physical Review Letters 86, 2416-9 (2001).

3. Hebard, A. F. et al. Superconductivity at $18 \mathrm{~K}$ in potassium-doped C60. Nature 350, 600-601 (1991).

4. Hannay, N. B. et al. Superconductivity in Graphitic Compounds. Physical Review Letters 14, 225-6 (1965).

5. Weller, T. E., Ellerby, M., Saxena, S. S., Smith, R. P. \& Skipper, N. T. Superconductivity in the intercalated graphite compounds $\mathrm{C}_{6} \mathrm{Yb}$ and $\mathrm{C}_{6} \mathrm{Ca}$. Nature Physics 1, 39-41 (2005).

6. Emery, N. et al. Superconductivity of Bulk CaC . Physical Review Letters 95, 087003-4 (2005).

7. Novoselov, K. S. et al. Two-dimensional gas of massless Dirac fermions in graphene. Nature 438, 192-200 (2005).

8. Zhang, Y., Tan, Y. W., Stormer, H. L. \& Kim, P. Experimental observation of the quantum Hall effect and Berry's phase in graphene. Nature 438, 201-204 (2005).

9. Forbeaux, I., Themlin, J. M. \& Debever, J. M. Heteroepitaxial graphite on $6 \mathrm{H}-\mathrm{SiC}(0001)$ : Interface formation through conduction-band electronic structure. Phys. Rev. B 58, 16396-406 (1998).

10. Berger, C. et al. Electronic Confinement and Coherence in Patterned Epitaxial Graphene. Science 312, 1191-6 (2006).

11. DiVincenzo, D. P. \& Mele, E. J. Self-consistent effective-mass theory for intralayer screening in graphite intercalation compounds. Physical Review B 29, 1685-94 (1984).

12. $\mathrm{Xu}, \mathrm{S}$. M. et al. Energy Dependence of Electron Lifetime in Graphite Observed with Femtosecond Photoemission Spectroscopy. Phys. Rev. Lett. 76, 483-486 (1996).

13. Moos, G., Gahl, C., Fasel, F., Wolf, M. \& Hertel, T. Anisotropy of Quasiparticle Lifetimes and the Role of Disorder in Graphite from Ultrafast Time-Resolved Photoemission Spectroscopy. Phys. Rev. Lett. 87, 267402 (2001).

14. Fröhlich, H. Superconductivity in metals with incomplete inner shells. Journal of Physics C: Solid State Physics 1, 544-548 (1968).

15. Ruvalds, J. Plasmons and high-temperature superconductivity in alloys of copper oxides. Phys. Rev. B 35, 8869-72 (1987).

16. Uchoa, B. \& Castro Neto, A. Superconductivity in metal coated graphene. cond-mat, 060815 (2006).

17. Wallace, P. R. The Band Theory of Graphite. Physical Review 71, 622-34 (1947).

18. Strocov, V. N. et al. Photoemission from graphite: Intrinsic and self-energy effects. Phys. Rev. B 64, 075105 (2001).

19. Rollings, E. et al. Synthesis and characterization of atomically thin graphite films on a silicon carbide substrate. Journal of Physics and Chemistry of Solids 67, 2172-2177 (2006).

20. Ohta, T., Bostwick, B., Seyller, T., Horn, K. \& Rotenberg, E. Controlling the Electronic Structure of Bilayer Graphene. Science 313, 951-954 (2006).

21. Sugawara, K., Sato, T., Souma, S., Takahashi, T. \& Suematsu, H. Fermi surface and edge-localized states in graphite studied by high-resolution angle-resolved photoemission spectroscopy. Phys. Rev. B 73, 045124 (2006).

22. Zhou, S. Y. et al. First direct observation of Dirac fermions in graphite. Nat Phys 2, 595-599 (2006).

23. Emtsev, K. et al. Initial stages of the graphite-SiC(0001) interface formation studied by photoelectron spectroscopy. cond-mat, 0609383 (2006).

24. Zhou, S. Y., Gweon, G. H. \& Lanzara, A. Low energy excitations in graphite: the role of dimensionality and lattice defects. Annals of Physics 321, 1730-46 (2006).

25. Shirley, E., Terminello, L., Santoni, A. \& Himpsel, F. J. Brillouin-zone-selection effects in graphite photoelectron angular distributions. Phys. Rev. B 51, 13614-22 (1995). 
26. Bennich, P. et al. Photoemission study of K on graphite. Physical Review B 59, 8292-304 (1999).

27. Kaminski, A. \& Fretwell, H. M. On the extraction of the self-energy from angle-resolved photoemission spectroscopy. New Journal of Physics 7, 98 (2005).

28. Kordyuk, A. A. et al. Bare electron dispersion from experiment: Self-consistent self-energy analysis of photoemission data. Physical Review B (Condensed Matter and Materials Physics) 71, 214513-11 (2005).

29. Valla, T., Fedorov, A. V., Johnson, P. D. \& Hulbert, S. L. Many-body effects in angle-resolved photoemission: quasiparticle energy and lifetime of a Mo(110) suface state. Phys. Rev. Lett. 83, 2085-8 (1999).

30. Hengsberger, M., Purdie, D., Segovia, P., Garnier, M. \& Baer, Y. Photoemission study of a strongly coupled electron-phonon system. Phys. Rev. Lett. 83, 592-5 (1999).

31. Rotenberg, E., Schaefer, J. \& Kevan, S. D. Coupling between Adsorbate Vibrations and an Electronic Surface State. Phys. Rev. Lett. 84, 2925-8 (2000).

32. Valla, T. et al. Evidence for Quantum Critical Behavior in the Optimally Doped Cuprate $\mathrm{Bi}_{2} \mathrm{Sr}_{2} \mathrm{CaCu}_{2} \mathrm{O}_{8+\alpha}$. Science 285, 2110-3 (1999).

33. Lanzara, A. et al. Evidence for ubiquitous strong electron-phonon coupling in high-temperature superconductors. 412, 510-514 (2001).

34. Vitali, L., Schneider, M. A., Kern, K., Wirtz, L. \& Rubio, A. Phonon and plasmon excitation in inelastic electron tunneling spectroscopy of graphite. Phys. Rev. B 69, 121414 (2004).

35. Grimvall, G. The Electron-Phonon Interaction in Metals (North Holland Publishing Company, Amsterdam, 1981).

36. Calandra, M. \& Mauri, F. Theoretical Explanation of Superconductivity in $\mathrm{C}_{6} \mathrm{Ca}$. Phys. Rev. Lett. 95, 237002 (2005).

37. Castro Neto, A. Charge Density Wave, Superconductivity, and Anomalous Metallic Behavior in 2D Transition Metal Dichalcogenides. Phys. Rev. Lett. 86, 4382-5 (2001).

38. Jensen, E., Bartynski, R. A., Gustafsson, T. \& Plummer, E. W. Distortion of an Unoccupied Band in Be by the Electron-Plasmon Interaction. Physical Review Letters 52, 2172-5 (1984).

39. Hawrylak, P. Effective mass and lifetime of electrons in a layered electron gas. Phys. Rev. Lett. 59, 485-8 (1987).

40. Luk'yanchuk, I. \& Kopelevich, Y. Phase Analysis of Quantum Oscillations in Graphite. Phys. Rev. Lett. 93, 166402 (2004).

41. Hamada, N., Sawada, S. \& Oshiyama, A. New One-dimensional Conductors: Graphitic Microtubules. Phys. Rev. Lett. 68, 1579-81 (1992).

42. Ando, T., Nakanishi, T. \& Saito, R. Berry's Phase and Absence of Back Scattering in Carbon Nanotubes. J. Phys. Soc. Jpn. 67, 2857-2862 (1998).

43. Li, Z. Y., Hock, K. M., Palmer, R. E. \& Annett, J. F. Potassium-adsorption-induced plasmon frequency shift in graphite. Journal of Physics: Condensed Matter 3, S103-S106 (1991). 